\title{
Effect of diatomaceous earth on thermoelectric elements using multi-walled carbon nanotubes
}

\author{
Kosei Hasuike, Dang Trang Nguyen, and Kozo Taguchi \\ Department of Electrical and Electronic Engineering, Ritsumeikan university, Kusatsu, Shiga, \\ Japan
}

\begin{abstract}
Thermoelectric power generation is one of the expected new renewable energy in the future. However, the power generation capacity of thermoelectric devices is poor. In this research, we focused on utilizing diatomaceous earth to improve the performance of thermoelectric devices because it has low thermal conductivity. Our thermoelectric devices based on multi-walled carbon nanotube and diatomaceous earth have improved the open-circuit voltage about $30 \%$ compared with the conventional thermoelectric devices based on multi-walled carbon nanotube only.
\end{abstract}

Key words: thermoelectric elements, diatomaceous earth, multi-walled carbon nanotubes, renewable energy

\section{INTRODUCTION}

Thermoelectric conversion that can convert direct heat to electric power is attracting attention as a power generation technology without carbon dioxide emission and without moving parts such as turbine. Functional thermoelectric devices can generate electricity anywhere there is a heat source. Conversely, they can be used as a cooling device without the use of pump or fluid by supplying current. Maximizing the effectiveness of the thermoelectric module, which restricts their applications in both electricity generation and cooling device, are of principle importance.

Superior thermoelectric devices must have high thermoelectric figure index and low cost. The efficiency of thermoelectric devices is related to the thermoelectric figure of merit, ZT, defined as $\mathrm{S}^{2} \sigma \mathrm{T} / \mathrm{K}$, where $\mathrm{S}$ denotes the Seebeck coefficient, $\sigma$ is the electrical conductivity, $\mathrm{k}$ is the thermal conductivity, and $\mathrm{T}$ is the absolute temperature. Good thermoelectric materials must have a high Seebeck coefficient for enhanced thermoelectricity, low electrical resistivity to minimize Joule heating, and low thermal conductivity to sustain large temperature gradient [1]. Currently, many thermoelectric materials are inorganic semiconductors. However, some thermoelectric semiconductor materials are not practical because they are highly toxic and expensive. Therefore, we focused on organic materials. The organic material element is expected to promote the thermoelectric device. Further among organic materials, we are interested in Carbon nanotubes (CNT). There are several types of CNT. Mainly, CNT is categorized as singlewalled carbon nanotube (SWCNT) and multi-walled carbon nanotube (MWCNT). CNT has various characteristics, for example: thin and robust, passing electricity and conveying heat well.

CNT has many desirable characteristics from the viewpoint of thermoelectric conversion. Nevertheless, the ZT reported for bulk SWCNT has remained low. Because, CNT has high thermal conductivity and low Seebeck coefficient. For example, only about $60 \mu \mathrm{V} / \mathrm{K}$ of S was reported on the SWCNT film [2]

In this study, we focused on finding the possibility of thermoelectric devices using CNTs. To make CNTs a good thermoelectric material, it is necessary to lower the thermal conductivity. Therefore, we utilized diatomaceous earth. Diatomaceous earth is a naturally occurring soft powder material made of fossils of unicellular algae called diatoms. The diatom skeleton is mainly made of amorphous silica. Diatomaceous earth has a low thermal conductivity $(\sim 0.1 \mathrm{~W} / \mathrm{mK})$ because of its nanoporous structure, and recently has attracted attention as a candidate for the core insulation material in vacuum insulation panels of excellent performance [3-5].

Corresponding Author: Kozo Taguchi, Department of Electrical and Electronic Engineering, Ritsumeikan University, 1-1-1 NojiHigashi, Kusatsu, Shiga 525-8577, Japan, email: taguchi@se.ritsumei.ac.jp 
We pursued simple and high-performance thermoelectric devices. For this reason, we used multi-

walled carbon nanotube (MWCNT). MWCNT is much low-cost compared with SWCNT. The physicochemical and electronic properties of CNT are predominantly modulated by the sign and the density of charge carriers and thus tuned by ionic and molecular doping along with the charge-injections. Electrochemical doping/dedoping also changes the property of charge carriers. i.e., the Seebeck coefficient of CNT changes from positive ( $\mathrm{p}$ type) to negative (n-type). Polyethyleneimine (PEI) is also one of the chemicals that change properties. PEI donates electrons to MWCNT and makes it possible to convert p-type nanotubes to n-type [6]. In this research, we selected diatomaceous earth to further improve the performance of the thermoelectric device using MWCNT.

\section{EXPERIMENT}

To prepare the MWCNT/diatomaceous earth dispersions, scale measured quantities of diatomaceous earth powder and MWCNT were mixed together. In this experiment, the used MWCNT liquid has a concentration of $6 \%$ MWCNT. $20 \mathrm{ml}$ of this MWCNT liquid was sonicated for 30 minutes to improve dispersibility. Voltage characteristics and internal resistance of thermoelectric elements using MWCNT depend on the concentration [7]. First, the dispersion was being stirred for $24 \mathrm{~h}$. Second, the dispersion was applied to a filter paper to make the electrode. Then, as a drying process, natural drying was carried out for 24 h. Figure 1(a) shows images of MWCNT soaked into filter paper $(1 \mathrm{~cm} \times 3 \mathrm{~cm})$. Also, we prepared the mixture of the dispersion with PEI. Similarly, the mixture was being stirred for $24 \mathrm{~h}$. Subsequently, it was applied to a filter paper. Then, natural drying was carried out for 24 h.

Figure 1(b) and (c) show experimental setups, where a thermoelectric device has been measured voltage when heat was applied. We measured each sample device that MWCNT/diatomaceous earth and MWCNT/ diatomaceous earth containing PEI. Then, we coupled each device to copper sheet. Because, copper has low Seebeck coefficient, it does not affect the measurement. Also, characteristics of joining MWCNT/diatomaceous earth to MWCNT/ diatomaceous earth containing PEI was measured. Since this structure was constructed with p-type and n-type, a higher voltage can be obtained. When the junction part was applied to heat, the voltage increased due to a temperature difference from room temperature.

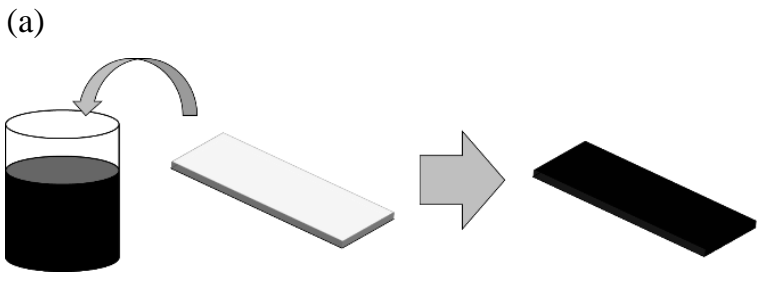

(b)

(c)
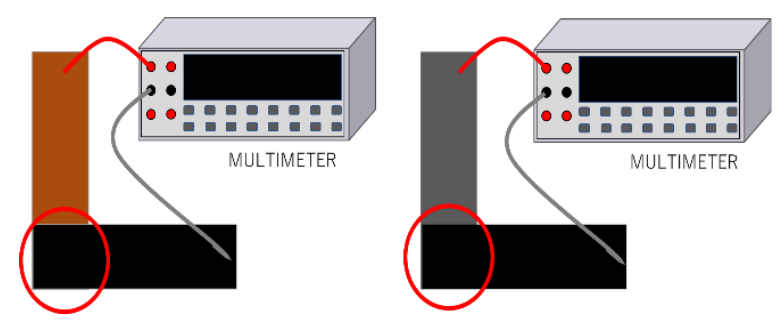

Figure 1. Structure of thermoelectric device and system of measurement. (a) Samples using in this experiment were filter paper $(1 \mathrm{~cm} \times 3 \mathrm{~cm})$ applied MWCNT/diatomaceous earth and MWCNT/diatomaceous earth containing PEI. (b) Composition when measuring characteristics of individual device. This device was composed of copper sheet connecting to MWCNT/diatomaceous earth or MWCNT/diatomaceous earth containing PEI. (c) Composition when measuring characteristic of joining MWCNT/diatomaceous earth with MWCNT/diatomaceous earth containing PEI (red circle shows heat applied area).

\section{RESULT AND DISCUSS}

\section{Influence of diatomaceous earth on p-type thermoelectric devices based on MWCNT}

MWCNT is an inexpensive alternative material with poorer electrical characteristics than SWCNT (about 50 to 100 times cheaper) $[8,9]$. In the thermoelectric element using MWCNT, the electric conductivity changes several orders of magnitude depending on the concentration of MWCNT. Figure 2 shows the electrical properties of thermoelectric devices using MWCNT and diatomaceous earth. The electrical characteristics of the MWCNT concentration of $6 \%$ was $1.15 \mathrm{mV}$ (opencircuit voltage) when heated at $150{ }^{\circ} \mathrm{C}$. The thermoelectric device with $0.2 \mathrm{~g}$ diatomaceous earth 
added to MWCNT was $1.26 \mathrm{mV}$ when heated at $150{ }^{\circ} \mathrm{C}$. The voltage of the thermoelectric device using MWCNT with $0.4 \mathrm{~g}$ diatomaceous earth was $1.55 \mathrm{mV}$ when heated at $150{ }^{\circ} \mathrm{C}$. The thermoelectric voltage has increased more than $30 \%$ by adding $0.4 \mathrm{~g}$ diatomaceous earth.

Based on these results, it can be confirmed that the addition of diatomaceous earth to MWCNT improved the electrical properties of thermoelectric devices based on MWCNT. This was attributed to the low thermal conductivity of diatomaceous earth because it is made of the diatom skeleton which is mainly composed of amorphous silica with nanoporous structure.

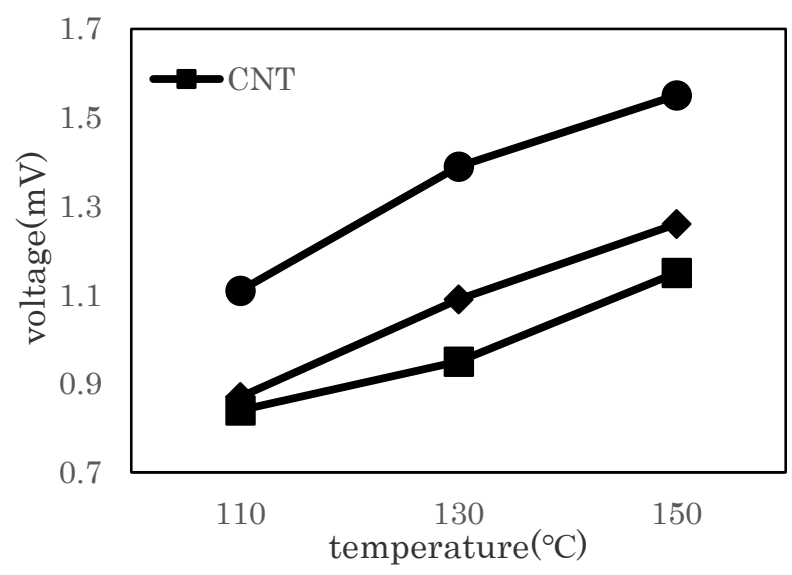

Figure 2. Relationship between electrical characteristics of thermoelectric devices using MWCNT and diatomaceous earth.

\section{Influence of diatomaceous earth on n-type thermoelectric devices based on MWCNT/PEI composite}

Several methods have been demonstrated to produce air stable n-type carbon nanotubes. For example, manufacturing passive structure around the tube to prevent oxygen doping, investing viologens for redox, and the application of metal electrodes with low work functions [10-12]. The simpler production method has also been demonstrated where the physical adsorption of branched polyethyleneimine (PEI) onto carbon nanotubes results in a conversion of the conductivity properties from p-type to n-type [13]. The amine groups are responsible to donate electron for which converts CNT into n-type semiconductors.

We have proposed a thermoelectric device using $\mathrm{p}$ type MWCNT/diatomaceous earth composite (above). In this part, the other type was made by adding PEI to convert the MWCNT conductivity from p-type to ntype. We measured electrical characteristics obtained by adding diatomaceous earth to the MWCNT/PEI composite as shown in Figure 3. By adding $10 \mathrm{ml}$ PEI to MWCNT, the conductivity was converted from $\mathrm{p}$ type to n-type (polarity changed from positive to negative). In addition, by adding $0.4 \mathrm{~g}$ diatomaceous earth to the MWCNT/PEI composite, thermoelectric voltage was improved from $-0.9 \mathrm{mV}$ to $-1.3 \mathrm{mV}$, about $44 \%$.

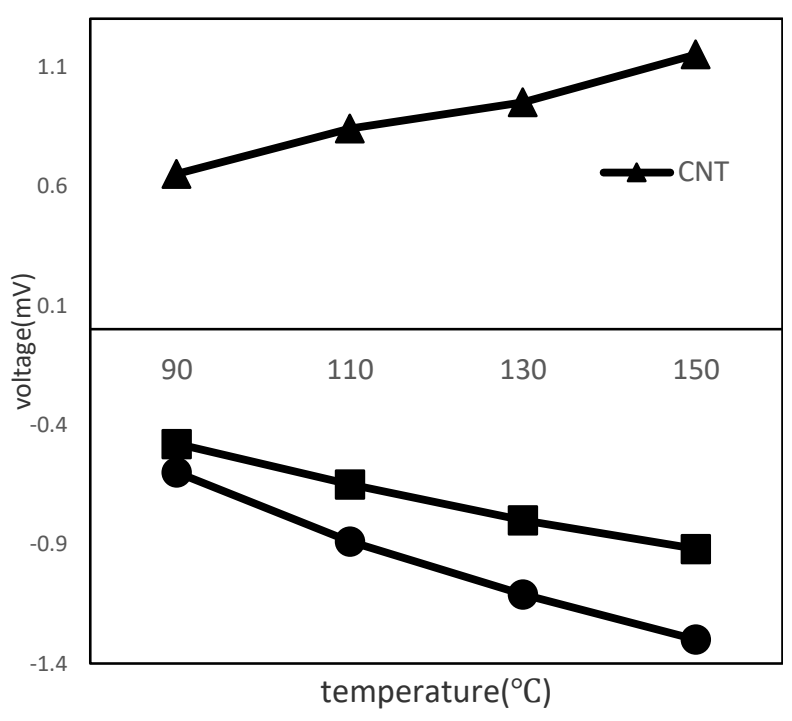

Figure 3. Electrical characteristics of thermoelectric devices when diatomaceous earth and polyethyleneimine are mixed with MWCNT.

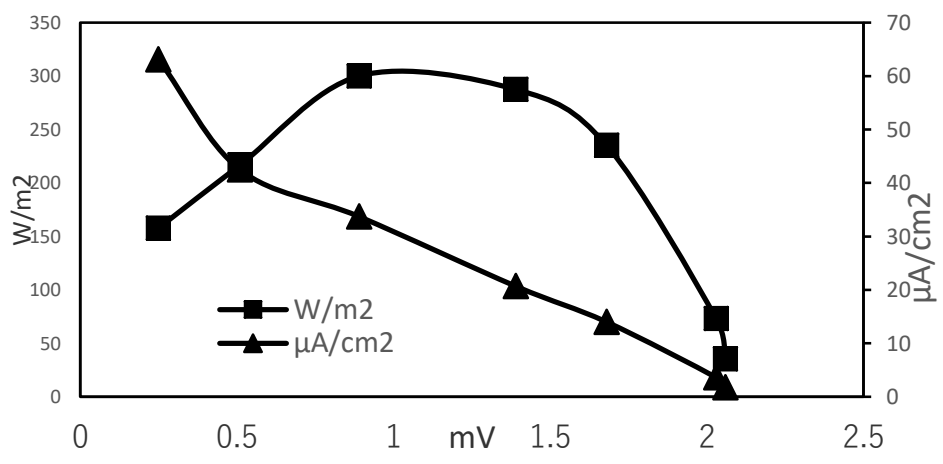

Figure 4. Power generation of fabricated n-type and ptype at $150^{\circ} \mathrm{C}$. 


\section{Improvement of thermoelectric devices based on the combination of the p-type and n-type materials}

The p-type and n-type materials synthesised above were combined to make the two electrodes of the proposed thermoelectric device. The p-type thermoelectric material was $20 \mathrm{ml}$ MWCNT mixed with $0.4 \mathrm{~g}$ diatomaceous earth. The n-type thermoelectric material was $20 \mathrm{ml}$ MWCNT mixed with $10 \mathrm{ml}$ PEI and $0.4 \mathrm{~g}$ diatomaceous earth. Heat is applied to this thermoelectric cell at $150{ }^{\circ} \mathrm{C}$. Figure 4 shows the power generation and current density output characteristics of this device as a function of discharging voltage measured at external resistors of $3.3,10,22,56,100$, $470,1000 \Omega$, respectively. The maximum output power density is $300 \mathrm{Wm}^{-2}$ measured against the working surface area of the substrate where the n-type and ptype thermoelectric materials attached.

\section{CONCLUSION}

We have confirmed that it is possible to improve ZT by lowering thermal conductivity by mixing diatomaceous earth with thermoelectric materials based on MWCNT. Furthermore, the thermoelectric device using MWCNT/PEI composite with diatomaceous earth acted as an n-type thermoelectric device. We confirmed the improvement of power density by the addition of diatomaceous earth. MWCNT makes it possible to manufacture thermoelectric devices that are much cheaper than SWCNT. In addition, it is important to improve performance by optimizing the composition of the composite materials and studying the effect of other dopants. Further studies on miniaturization of device, decrease in thermal conductivity, improvement of conductivity, and improvement of Seebeck coefficient are necessary.

\section{REFERENCES}

[1] M.S. Dresselhaus, G. Chen, M.Y. Tang, R.G. Yang, H. Lee, D.Z. Wang, et al., New directions for low-dimensional thermoelectric materials, Adv. Mater. 19 (8) (2007), pp. 1043-1053.

[2] J. Hone, I. Ellwood, M. Muno, A. Mizel, M. L. Cohen, A. Zettl, A. G.Rinzler, and R. E. Smalley, Phys. Rev. Lett. 80, 1042 (1998).

[3] J. Fricke, U. Heinemann, H.P. Ebert, Vacuum insulation panels-from research tomarket, Vacuum 82 (2008), pp. 680-690.

[4] J. Fricke, H. Schwab, U. Heinemann, Vacuum insulation panels - exciting thermal properties and most challenging applications, Int. J. Thermophys. 27 (2006), pp. 1123-1139.

[5] B. Chang, L. Zhong, M. Akinc, Low cost composites for vacuum insulation core material, Vacuum 131 (2016), pp. 120-126.

[6] Dallas D. Freeman, Kyungwho Choi, Choongho $\mathrm{Yu}, \mathrm{N}-\mathrm{Type}$ Thermoelectric Performance of Functionalized Carbon Nanotube-Filled Polymer Composites, Plos one (2012).

[7] Ruben Sarabia-Riquelmea, John Craddocka, E. Ashley Morrisa, David Eatona, Rodney Andrewsa, John Anthonyb, Matthew C. Weisenbergera, Synthetic Metals 225 (2017), pp. 86-92

[8] G.J. Snyder, E.S. Toberer Complex

Thermoelectric materials Nat. Mater., 7 (2) (2008), pp. 105-114

[9] O. Bubnova, Z.U. Khan, A. Malti, S. Braun, M. Fahlman, M. Berggren, et al. Optimization of the thermoelectric figure of merit in the conducting polymer poly (3, 4-ethylenedioxythiophene) Nat. Mater., 10 (6) (2011), pp. 429-433

[10] Kaminishi D, Ozaki H, Ohno Y, Maehashi K, Inoue $\mathrm{K}$, Air-stable n-type carbon nanotube field-effect transistors with si3n4 passivation films fabricated by catalytic chemical vapor deposition. Appl Phys Lett 86 (2005).

[11] Kim SM, Jang JH, Kim KK, Park HK, Bae JJ, et al., Reductioncontrolled viologen in bisolvent as an environmentally stable n-type dopant for carbon nanotubes. J Am Chem Soc 131 (2008), pp. 327331.

[12] Zhang Z, Liang X, Wang S, Yao K, Hu Y, et al., Doping-free fabrication of carbon nanotube based ballistic cmos devices and circuits. Nano Lett 7 (2007), pp. 3603-3607

[13] Shim M, Javey A, Kam NWS, Dai H, Polymer functionalization for airstable n-type carbon nanotube field-effect transistors. J Am Chem Soc (2001), pp. 11512-11513. 